\title{
Laser interstitial thermal therapy for newly diagnosed and recurrent glioblastoma
}

\author{
Jonathan G. Thomas, MD, ${ }^{1}$ Ganesh Rao, MD, ${ }^{1}$ Yvonne Kew, MD, PhD, ${ }^{2}$ and Sujit S. Prabhu, MD ${ }^{1}$ \\ 1'Department of Neurosurgery, University of Texas MD Anderson Cancer Center; and 2Department of Neurology, Houston \\ Methodist Hospital, Houston, Texas
}

\begin{abstract}
OBJECTIVE Glioblastoma (GBM) is the most common and deadly malignant primary brain tumor. Better surgical therapies are needed for newly diagnosed GBMs that are difficult to resect and for GBMs that recur despite standard therapies. The authors reviewed their institutional experience of using laser interstitial thermal therapy (LITT) for the treatment of newly diagnosed or recurrent GBMs.

METHODS This study reports on the pre-LITT characteristics and post-LITT outcomes of 8 patients with newly diagnosed GBMs and 13 patients with recurrent GBM who underwent LITT.

RESULTS Compared with the group with recurrent GBMs, the patients with newly diagnosed GBMs who underwent LITT tended to be older ( 60.8 vs 48.9 years), harbored larger tumors (22.4 vs $\left.14.6 \mathrm{~cm}^{3}\right)$, and a greater proportion had IDH wild-type GBMs. In the newly diagnosed GBM group, the median progression-free survival and the median survival after the procedure were 2 months and 8 months, respectively, and no patient demonstrated radiographic shrinkage of the tumor on follow-up imaging. In the 13 patients with recurrent GBM, 5 demonstrated a response to LITT, with radiographic shrinkage of the tumor following ablation. The median progression-free survival was 5 months, and the median survival was greater than 7 months.
\end{abstract}

CONCLUSIONS In carefully selected patients with recurrent GBM, LITT may be an effective alternative to surgery as a salvage treatment. Its role in the treatment of newly diagnosed unresectable GBMs is not established yet and requires further study.

http://thejns.org/doi/abs/10.3171/2016.7.FOCUS16234

KEY WORDS glioblastoma; GBM; recurrence; laser interstitial thermal therapy; LITT

$\mathrm{G}$ LIOBLASTOMA (GBM) is the most common malignant primary brain tumor. Due to lack of effective therapies, patients with newly diagnosed GBM have a median survival of only 14 months. ${ }^{26}$ In the most current treatment paradigm, GBMs are treated with a combination of maximal safe resection followed by chemotherapy and radiation therapy (RT), ${ }^{2,6}$ Based on data from large retrospective studies of GBM, it appears that a greater extent of resection is associated with improved survival. ${ }^{12,13,23}$ Extending from this, the concept that cytoreductive therapies positively impact outcome has received more widespread acceptance. Chemotherapy and RT without any surgical cytoreductive therapies are associated with worse outcomes. ${ }^{25,27}$ However, a significant number of GBMs up to $40 \%$ - are considered "unresectable" and are not amenable to aggressive resection. ${ }^{5}$ These patients have a worse overall median survival of 9.4 months. ${ }^{26}$ Unfortunately, the induction of a postoperative motor or language deficit is associated with a diminution of median survival by $3-4$ months, ${ }^{14}$ so attempting an aggressive resection on so-called unresectable tumors can result in patient harm in terms of both function and overall survival. For these patients with GBMs that are not amenable to resection, a cytoreductive intervention that respects and preserves the eloquent areas of the brain may help close the gap in survival, bringing it closer in line with patients who can undergo resection.

Laser interstitial thermal therapy (LITT) for percutaneous ablation of intracranial tumors has been explored since the late 1970s, but never received widespread adoption in

ABBREVIATIONS CCNU = 1-(2-chloroethyl)-3-cyclohexyl-1-nitrosourea; GBM = glioblastoma; KPS = Karnofsky Performance Scale; LITT = laser interstitial thermal therapy; $\mathrm{NIH}=$ National Institutes of Health; PFS = progression-free survival; RT = radiation therapy; TDT = thermal damage threshold; TMZ = temozolomide.

SUBMITTED June 1, 2016. ACCEPTED July 25, 2016.

INCLUDE WHEN CITING DOI: 10.3171/2016.7.FOCUS16234. 
clinical practice due to an inability to achieve tight control over the areas of ablation. ${ }^{15}$ However, the advent of MR thermography coupled with improved image guidance and laser technology has brought broader applications for LITT in the field of neurosurgery. The LITT procedure offers several features that make it attractive for the treatment of unresectable gliomas: 1) it is a percutaneous procedure, so wound healing rarely interferes with chemotherapy or RT; 2) it can be combined with functional preoperative imaging studies such as diffusion tensor imaging to ensure that eloquent fiber tracts or cortex remain outside the ablation zone; and 3) the minimal blood loss and fluid shifts during the surgery allow it to be performed in patients who may not be candidates for an open resection due to comorbidities.

Additionally, LITT may be a good option for focal recurrences of GBMs that have previously undergone resection. Although the consensus on the best treatment of recurrent disease is not as solid as with newly diagnosed disease, multiple retrospective studies support the excision of resectable recurrences. ${ }^{17,19,28}$ However, with recurrent disease, issues of wound healing and the patient's functional status can hamper attempts of total resection. Moreover, recurrences often occur at sites close to functional or eloquent brain that were respected during the first surgery. Therefore, LITT may offer a cytoreductive option for focal GBM recurrences.

We report our experience at MD Anderson Cancer Center using LITT for treatment of both newly diagnosed "unresectable" GBMs and recurrent GBMs.

\section{Methods}

We retrospectively reviewed patients with a diagnosis of GBM, either newly diagnosed or recurrent, who underwent LITT at MD Anderson Cancer Center with at least 6 months of follow-up. Institutional review board approval was obtained. Newly diagnosed GBM included patients who were either diagnosed at the time of the procedure or those who had been diagnosed previously with biopsy sampling or a subtotal resection or debulking within 1 month of the LITT procedure, and who had not yet received any oncological treatments. Patients with recurrent GBM included those treated previously with total or subtotal resections, chemoradiation, and any other additional therapies who eventually underwent LITT for progression. Patients who had undergone a biopsy only and then were treated with chemoradiation prior to LITT were also included in this group. Due to the nature of referral patterns at our institution, the majority of the patients in the recurrent GBM group received their initial oncological care elsewhere. Preoperative clinical data that were collected included Karnofsky Performance Scale (KPS) score, previous chemotherapies and RTs, IDHI and other mutational status when available, and baseline neurological deficits. When the diagnosis was made at another institution, pathology slides were reviewed at our institution to confirm the diagnosis.

The LITT procedures were performed using an operating room suite with an integrated 1.5-T open-bore intraoperative MRI unit. One of 2 primary surgeons (S.P. or G.R.) performed the surgery with either the Medtronic Visualase or Monteris NeuroBlate systems. The initial cases were all done using the Visualase system, whereas the later cases were done using the NeuroBlate system. Laser and catheter placement was aided with intraoperatively acquired registration MRI scans and the Brainlab Varioguide system. In the few cases in which multiple trajectories were needed, the ClearPoint navigation system was used. In each case, the enhancing portion of the tumor was targeted, and ablation proceeded until the estimated irreversible damage zone covered the entire target, or until this zone encroached on densely functional and/or eloquent regions of the brain. In cases with diffuse contrast-enhancing disease, the bulkiest central part of the lesion was targeted. For tumors near cortical and subcortical motor areas, we used radiographic identification of the motor strip and preoperative diffusion tensor imaging to identify the limits of ablation. For insular lesions, we ablated contrast-enhancing lesion medially up to the internal capsule. For lesions near potential speech areas (e.g., left frontal, left temporal, and left inferior parietal lobule in right-handed persons), we attempted to keep the ablation zone confined to the enhancing areas. Optic radiations and visual cortex generally did not limit the extent of ablation. Postoperatively, patients were placed on a slow dexamethasone taper.

Postoperative data that were reviewed and collected included the presence of any new neurological deficits after LITT, time to clinical and/or radiographic progression, postprocedural chemotherapy or radiation regimens, and overall survival. Postoperative MRI studies were performed on the day of or 1 day after the procedure, 1 month after the procedure, and then in most cases every 2-4 months thereafter, although for international patients there was sometimes limited access to follow-up imaging. Preoperative and postoperative contrast-enhanced MR images were reviewed to assess preoperative tumor characteristics, radiographic response, and measurement of preoperative and serial postoperative volumes. Segmentations for calculation of contrast-enhancing tumor volume were performed using the Brainlab iPlan software. Patients who demonstrated an objective decrease in the volume of contrast-enhancing tumor in each MRI session from the first postoperative study to 6 months postprocedure and who had no evidence of clinical progression were designated "responders." For recurrent GBM cases, the National Institutes of Health (NIH) Recurrent GBM Scale score was calculated, with 1 point each for KPS score of $\leq$ 80 , tumor size $\geq 50 \mathrm{~cm}^{3}$, and involvement of $\geq 2$ eloquent regions of the brain.

\section{Results}

\section{Newly Diagnosed Group}

Between August 2013 and November 2015, there were 8 newly diagnosed patients with GBM and 13 with recurrent GBM who had been treated with LITT (Table 1). In the newly diagnosed group, the average age was 61 years, the median age was 58 years, and the median KPS score was 85 . Genetic analysis was available in 4 of the 8 patients, and none had a GBM with the IDH mutation. Six 
TABLE 1. Data and outcomes pre- and post-LITT for 21 patients with GBM

\begin{tabular}{|c|c|c|c|}
\hline Characteristic & Newly Diagnosed & Recurrent & $\begin{array}{l}\text { Recurrent } \\
\text { Responder }\end{array}$ \\
\hline No. of patients & 8 & 13 & 5 \\
\hline Avg age & $60.8 \mathrm{yrs}$ & $48.9 \mathrm{yrs}$ & $50.2 \mathrm{yrs}$ \\
\hline Median mos from diagnosis & 1 & 16 & 11 \\
\hline Median KPS score & 85 & 80 & 80 \\
\hline IDH1 mutant* & $0 \%$ & $40 \%$ & $60 \%$ \\
\hline Multifocal lesion & $75 \%$ & $69 \%$ & $80 \%$ \\
\hline Periventricular tumor & $88 \%$ & $46 \%$ & $60 \%$ \\
\hline Lesion in eloquent area & $63 \%$ & $62 \%$ & $100 \%$ \\
\hline Location & 5 "butterfly," 2 insular, 1 thalamic & $\begin{array}{c}3 \text { motor, } 3 \text { speech, } 1 \text { rt temporal, } 2 \\
\text { splenium, } 2 \text { cingulate, } 2 \text { insular }\end{array}$ & $\begin{array}{l}2 \text { motor, } 2 \text { insular, } \\
1 \text { speech }\end{array}$ \\
\hline Patients w/ neuro deficit & $50 \%$ & $77 \%$ & $60 \%$ \\
\hline Avg preop tumor vol $\left(\mathrm{cm}^{3}\right)$ & 22.4 & 14.6 & 8.5 \\
\hline Avg NIH Recurrent GBM Scale score & NA & 1.1 & 1.2 \\
\hline Complications & 1 functional decline, transfer to hospice & 1 status epilepticus w/ functional decline & None \\
\hline Median $\%$ change in vol at 3 mos post-LITT & NA† & $68 \%$ & $-53 \%$ \\
\hline Median PFS & $2 \mathrm{mos}$ & 5 mos & NA \\
\hline Median survival after LITT & 8 mos (range $0-15$ mos) & $>7 \mathrm{mos}$ & NA \\
\hline
\end{tabular}

Avg = average; $N A=$ not available; neuro = neurological.

* Percentage of IDH mutants in patients in whom mutational analysis was done.

$\dagger$ Due to high rates of clinical decline, only 4 patients had radiographic follow-up.

$\ddagger$ Four patients still alive; 3 of them still progression free.

patients had multilobar involvement, 5 had bihemispheric corpus callosum tumors, 2 had involvement of insula, and 1 had thalamic/basal ganglia involvement. Seven of 8 patients had ependymal wall tumor involvement. Two patients had previously undergone craniotomy within 1 month of LITT, with extirpation of "resectable" portions of the tumor. The mean preoperative volume was 22.4 $\mathrm{cm}^{3}$. Successful ablative coverage of the entire contrastenhancing target was estimated to have been achieved in 4 of 8 cases at the time of the procedure. Postprocedure, no patients suffered any immediate new neurological deficits. However, 1 patient with a preoperative KPS score of 60 suffered a decline in functional status postoperatively, and the family wished to transition this patient to palliative care. The other 7 patients received standard postoperative concurrent temozolomide (TMZ) and RT. None of the patients were deemed "responders," and all showed either radiographic or clinical progression after surgery. Figure 1 shows imaging findings in a patient who underwent LITT for a newly diagnosed "butterfly" GBM. Despite successful ablation, there was no radiographic evidence of disease shrinkage. He did, however, live for 12 months after the procedure. In the newly diagnosed group, the median progression-free survival (PFS) was 2 months, and the median survival from procedure was 8 months.

\section{Group With Recurrent GBM}

In our group of 13 patients with recurrent GBM, the average age was 49 years, the median age was 50 years, and the median KPS score was 80 . The procedure was performed a median of 16 months from initial diagnosis. One patient had undergone a previous biopsy, 5 had received previous subtotal resection, and 6 had received previous total resection. Twelve patients had undergone RT previously, 12 had received TMZ previously, 1 had undergone stereotactic radiosurgery previously, and 2 had received additional bevacizumab chemotherapy. Five of the 11 patients in whom mutational analysis was available had IDHI mutations, and 4 tumors were secondary GBMs that had progressed from an identified lower-grade tumor. At the time of the LITT procedure, most patients had intermediate NIH Recurrent GBM Scale scores; 3 patients had a score of 0,7 had a score of 1 , and 3 had a score of 2 . The mean preoperative tumor volume was $14.6 \mathrm{~cm}^{3}$. Sixty-nine percent of the tumors were multifocal, with $62 \%$ in eloquent brain and $46 \%$ involving the ependymal wall. Postoperatively, there were no new focal neurological deficits. One patient with a large $65-\mathrm{cm}^{3}$ tumor suffered from status epilepticus in the postoperative recovery period. His seizures were eventually controlled, but he then transferred to hospice rather than receive further oncological care. The other 12 patients received adjuvant therapies, including TMZ, 1-(2-chloroethyl)-3-cyclohexyl1-nitrosourea (CCNU; i.e., lomustine), and bevacizumab. The median time to radiographic or clinical progression was 5 months, and the median overall survival was greater than 7 months from the LITT procedure (7 patients are still alive).

\section{Recurrent Responders Group}

Among the 5 "recurrent responders" who had a radiographic shrinkage of disease postablation, 4 patients had an NIH Recurrent GBM Scale score of 1 , and 1 patient had a score of 2 . Compared with the 8 nonresponders, the 

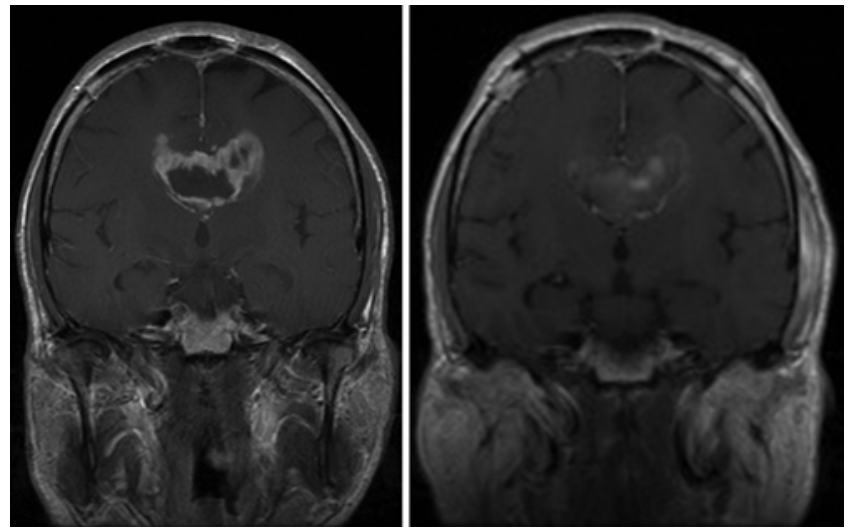

FIG. 1. Preoperative (left) and immediately postoperative (right) coronal T1-weighted contrast-enhanced MRI studies obtained in a 52-year-old man who underwent LITT for a newly diagnosed "butterfly" GBM. He had good ablative coverage of the lesion, but it failed to demonstrate shrinkage on subsequent MR images. He returned to work as a college professor but died 12 months after the procedure.

average preoperative tumor volume was smaller $\left(8.5 \mathrm{~cm}^{3}\right.$ vs $\left.17.4 \mathrm{~cm}^{3}\right)$ and there was higher proportion of IDHI mutants (60\% vs $13 \%)$, but the median KPS score was similar (80 vs 85). Among the 5 responders, 4 are still alive with a median time to radiographic progression of 9 months. In 3 of these 5 patients the tumors had $I D H 1$ mutations and progressed from a previously diagnosed lower-grade glioma. Two of 5 patients received the adjuvant combination of CCNU and bevacizumab within 3 months of surgery, whereas the other 3 received no adjuvant therapies initially. Figure 2 shows preoperative, immediate postablation, and 3-month follow-up MR images of a "responder" patient who underwent LITT for recurrence of GBM in the left parietal lobe.

\section{Discussion}

The consensus optimal therapy for newly diagnosed GBM is maximal safe resection followed by chemoradiation. However, in patients with so-called unresectable GBMs, optimal therapy is currently biopsy only followed by chemoradiation. Unfortunately, these patients have a survival disadvantage compared with patients who are able to undergo a more aggressive resection. Whether this worse prognosis is due to intrinsically more malignant biological behavior in these unresectable tumors, which tend to be more diffuse, or whether it is due to lack of effective direct cytoreductive therapies has not been fully clarified. If it is the latter, LITT may provide a useful therapeutic option for these patients. Unlike stereotactic radiosurgery, another focal therapy that can treat difficult-to-access targets, the tumor cell death after LITT is immediate and without a latency period.

Our 8 patients with newly diagnosed GBMs had unresectable tumors, with 5 exhibiting a bihemispheric, socalled butterfly spread, and the other 3 involving insula or deep nuclei. Four patients had preoperative focal neurological deficits. Only 1 patient was younger than 50 years, and in the 4 patients who received mutational analysis, none tested positive for $I D H 1$ mutation. In this group, no patients qualified as LITT responders with shrinkage of the disease in postprocedure surveillance MRIs, and it is unclear that the procedure altered the clinical outcome as compared with standard chemotherapy and RT. The median time to radiographic progression was 1.5 months, and the median survival was 8 months, which is in line with what has been reported in patients who underwent biopsy and chemoradiation only. Patients with butterfly GBMs have a worse survival prognosis, with a median survival of only 3.5 months after biopsy and, for those who can undergo surgery, 5.1 months. ${ }^{3}$ At our institution, we usually perform surgery for eccentric corpus callosum GBMs associated with mass effect, but for those with more symmetrical disease and extensive invasive disease, we refrain from resection. The patients in this series therefore tended to be in this latter group. The youngest patient in the group (48 years old) remained alive with stable disease at 11 months, but his $I D H$ status was unknown. Importantly, there were no new neurological deficits induced, although 1 patient with a butterfly GBM and a preoperative KPS score of 60 who was experiencing rapid decline suffered mild functional decline after LITT and wished to be transferred to hospice rather than undergo further treatments.

There have been few studies of the treatment of newly diagnosed GBMs with LITT, with 5 studies including treatment of a mix of 40 WHO Grade III and IV newly

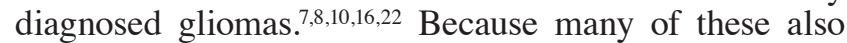
mixed in the results with those in recurrent gliomas, it is difficult to draw conclusions about the effectiveness of LITT for these lesions. Similar to our study, most of these patients with newly diagnosed high-grade gliomas were treated with LITT rather than surgery due to unresectable tumor location. The disappointing results in our series regarding the treatment of these unresectable GBMs probably reflect the limitations of a focal treatment for a diffuse disease as well as the more malignant biology in these cases. Most of these tumors displayed a more diffuse and invasive phenotype, with all but 1 being multilobar or bihemispheric. The contrast-enhancing portion of these tumors is only a small fraction of the actual tumor burden in these cases. Additionally, unresectability may be a surrogate for more malignant behavior-for example, in 1 study, $I D H$ wild-type gliomas were significantly less amenable to resection than $I D H$ mutant gliomas. ${ }^{1}$ Thus, these patients may be predisposed to a worse natural history even after effective cytoreduction.

Patients with GBMs are living longer than before, ${ }^{11}$ and coinciding with this, surgical treatment of GBM recurrences has become more commonplace. In a recent review of reoperation for GBM, 24 of 28 studies reported a survival benefit or improved functional status following reoperation and adjuvant treatments. Given the greater emphasis on cytoreduction and favorable retrospective data, repeat resection is often offered to carefully selected patients. The NIH Recurrent GBM Scale attempts to objectively predict which patients may receive the most benefit from a reoperation ${ }^{21}$ based on tumor involvement of eloquent brain, KPS score, and tumor volume. An alternative scoring system has also been introduced, which includes the unfavorable variable of ependymal involvement. ${ }^{20}$ However, rates of complications are significantly 

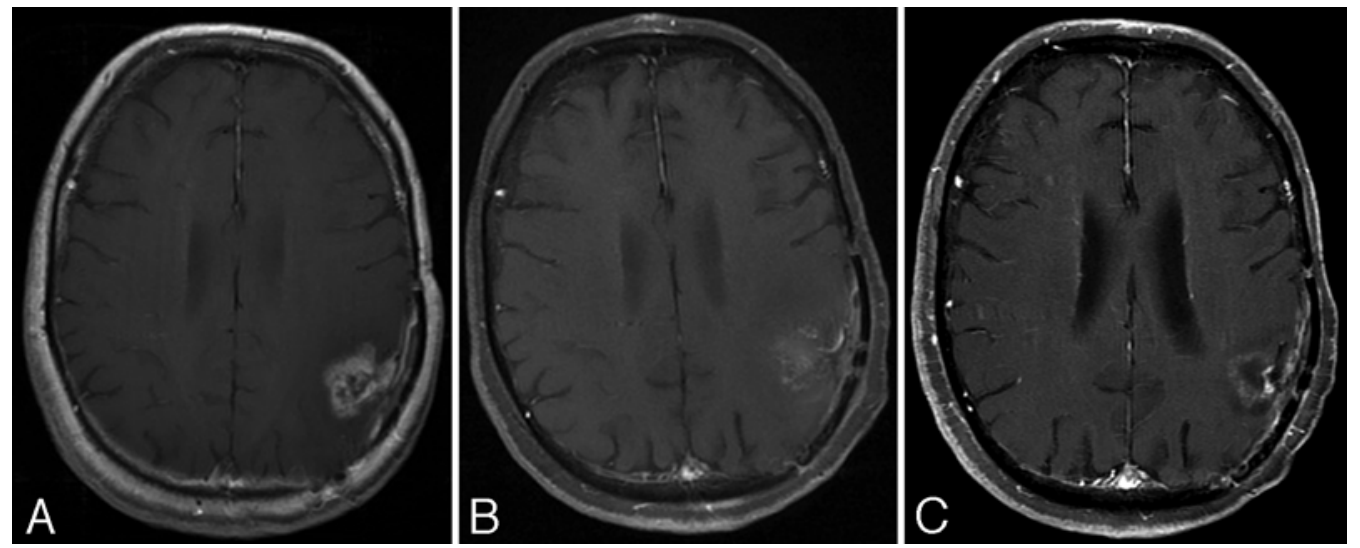

FIG. 2. Preoperative (A), immediately postoperative (B), and 3-month postoperative (C) axial T1-weighted contrast-enhanced MRI studies obtained in a 57-year-old woman who underwent LITT for a recurrent left parietal IDH wild-type GBM. She showed good response to LITT, with a $68 \%$ decrease in tumor volume, and remains progression free 7 months after the procedure.

higher (27\% vs $13 \%$ ) in second resections compared with the first resections, with an increased risk of infection and poor wound healing, and worse postoperative functional and neurological outcomes. ${ }^{4,9}$ Recurrences often occur at the edges of resection that border on eloquent regions. Additionally, there appears to be stepwise increase in the risk of wound issues (infection, CSF leakage, wound breakdown) with each successive surgery. ${ }^{9}$ Wound healing issues can be particularly devastating to these patients, who depend on further supplementary adjuvant therapies for tumor control. By minimizing wound healing issues, LITT could offer an attractive alternative way to surgically address GBM recurrences, even in areas accessible by repeat resection.

In our study, in comparison with the newly diagnosed GBM group, the 13 patients in the recurrent GBM group were younger, with an average age of 49 years. They harbored more indolent GBMs, with a median time from diagnosis to LITT of 16 months, and of the 10 patients in whom mutational analysis was available, 4 harbored IDHI mutant tumors. The tumors were generally smaller in the recurrent than in the newly diagnosed group. Although the preference at our institution is to resect accessible recurrent GBMs in patients who can tolerate it, in this group we treated 4 resectable lesions -2 deep cingulate gyrus recurrences and 2 noneloquent focal nodular recurrences-with LITT. Patients in this group had intermediate NIH Recurrent GBM Scale scores. The median overall survival after LITT was greater than 7 months (7 patients still alive), more than the 4.5 months predicted by the NIH Recurrent GBM Scale. ${ }^{21}$

Compared with nonresponding patients, patients who were responders had smaller tumors and were more likely to harbor an IDHI mutation (60\% had the mutation). Tumor size has previously been shown to affect ablation effectiveness. In 1 multiinstitutional study of LITT for highgrade gliomas, tumor volume $>10 \mathrm{~cm}^{3}$ was associated with worse PFS outcomes, even with good ablation coverage. ${ }^{16}$ Two of the responders in our study were receiving CCNU in the postoperative period when disease shrinkage was seen. This may be confounding, but it also may hint at a synergistic effect between LITT and chemotherapies. The
LITT procedure promotes breakdown of the blood-brain barrier in the peripheral zone of ablation, and this can be seen in immediate postoperative scans, which show the hazy contrast enhancement in that region..$^{18,24}$ This may potentiate the effects of chemotherapies by allowing easier entry across the blood-brain barrier, even with lipidsoluble drugs like CCNU. With a median overall survival and median PFS that compares favorably to most series of recurrent GBM, despite the fact that many of the tumors in our series were in deep or eloquent regions, and with 5 of 13 patients showing impressive radiographic shrinkage of disease, LITT may be a reasonable salvage therapy option in carefully selected patients with recurrent GBM.

In our series we had a favorable complication profile, with no new permanent neurological deficits, 1 case of worsening seizures, and 2 cases of worsening functional status ultimately proceeding to palliative care. No patient suffered superficial or deep wound infection or any significant intracranial hemorrhage. Whereas other studies usually include both WHO Grades III and IV gliomas in their series, our study focused only on GBMs because their behavior differs significantly from anaplastic astrocytoma and especially anaplastic oligodendrogliomas. Due to the more indolent course of Grade III gliomas, lengthy followup is necessary and it can be more difficult to see a treatment's effect on the natural history. With this in mind, we also included data on IDHI mutation status, which clearly helped identify the varying biological behavior of these tumors. We also separated newly diagnosed from recurrent GBMs. As our study showed, preoperatively these groups contained 2 very different populations and different tumor biological features. This discrepancy may be due in part to local referral patterns, but it is also likely that newly diagnosed GBMs treated with LITT will be "unresectable," with a more diffuse and invasive phenotype presaging more aggressive disease than in patients with recurrent GBMs previously treated with surgery who are healthy enough to warrant further aggressive treatment. Studies that do not separate these 2 groups of patients may have to be interpreted with caution.

However, our study has important weaknesses. Its retrospective nature limits the strength of any conclusions 
that can be drawn. The recurrent GBM group was clearly a carefully selected subgroup of patients with GBM, which curtails the generalizability of our findings. The small numbers of patients in the study precluded multivariate analysis, which is especially crucial in a retrospective analysis of patients with recurrent GBM in whom there is a high variability in previous treatments and adjuvant treatments. Additionally, ideally we would have stratified by genetic and mutational status, but although in the majority of patients these data were available, they were incomplete. Even in tumors that tested negative for the IDHI mutation, noncanonical IDHI mutations were not necessarily tested. Unfortunately, due to the referral patterns of the hospital, the initial biopsy or surgery was often done elsewhere, which limited further genetic and molecular analysis of the tumor. Additionally, follow-up radiographic data were not uniformly available, and we did not have data available on thermal damage threshold (TDT) lines with respect to tumor coverage. Adequate TDT coverage may play a role in enhancing PFS, ${ }^{16}$ and these TDT lines allow for a standardization of the ablation technique and easier comparisons with other series. Because we had switched between the Visualase and NeuroBlate systems, these were not available for review. And because these were our initial patients with GBM treated with LITT, a learning curve may have also been in play, with greater facility and effectiveness of technique achieved in the later cases compared with the earlier ones.

\section{Conclusions}

Laser interstitial thermal therapy may be an effective alternative to surgery as a salvage treatment in carefully selected patients with recurrent GBM. Its role in the treatment of newly diagnosed unresectable GBMs is not established yet and requires further study.

\section{References}

1. Beiko J, Suki D, Hess KR, Fox BD, Cheung V, Cabral M, et al: IDH1 mutant malignant astrocytomas are more amenable to surgical resection and have a survival benefit associated with maximal surgical resection. Neuro Oncol 16:81-91, 2014

2. Bush NAO, Chang SM, Berger MS: Current and future strategies for treatment of glioma. Neurosurg Rev [epub ahead of print], 2016

3. Chaichana KL, Jusue-Torres I, Lemos AM, Gokaslan A, Cabrera-Aldana EE, Ashary A, et al: The butterfly effect on glioblastoma: is volumetric extent of resection more effective than biopsy for these tumors? J Neurooncol 120:625-634, 2014

4. Chang SM, Parney IF, McDermott M, Barker FG II, Schmidt $\mathrm{MH}$, Huang W, et al: Perioperative complications and neurological outcomes of first and second craniotomies among patients enrolled in the Glioma Outcome Project. J Neurosurg 98:1175-1181, 2003

5. Fazeny-Dörner B, Wenzel C, Veitl M, Piribauer M, Rössler K, Dieckmann K, et al: Survival and prognostic factors of patients with unresectable glioblastoma multiforme. Anticancer Drugs 14:305-312, 2003

6. Fine HA, Dear KB, Loeffler JS, Black PM, Canellos GP: Meta-analysis of radiation therapy with and without adjuvant chemotherapy for malignant gliomas in adults. Cancer 71:2585-2597, 1993
7. Hawasli AH, Bagade S, Shimony JS, Miller-Thomas M, Leuthardt EC: Magnetic resonance imaging-guided focused laser interstitial thermal therapy for intracranial lesions: singleinstitution series. Neurosurgery 73:1007-1017, 2013

8. Hawasli AH, Kim AH, Dunn GP, Tran DD, Leuthardt EC: Stereotactic laser ablation of high-grade gliomas. Neurosurg Focus 37(6):E1, 2014

9. Hoover JM, Nwojo M, Puffer R, Mandrekar J, Meyer FB, Parney IF: Surgical outcomes in recurrent glioma: clinical article. J Neurosurg 118:1224-1231, 2013

10. Jethwa PR, Barrese JC, Gowda A, Shetty A, Danish SF: Magnetic resonance thermometry-guided laser-induced thermal therapy for intracranial neoplasms: initial experience. Neurosurgery 71 (1 Suppl Operative):133-145, 2012

11. Johnson DR, O'Neill BP: Glioblastoma survival in the United States before and during the temozolomide era. J Neurooncol 107:359-364, 2012

12. Lacroix M, Abi-Said D, Fourney DR, Gokaslan ZL, Shi W, DeMonte F, et al: A multivariate analysis of 416 patients with glioblastoma multiforme: prognosis, extent of resection, and survival. J Neurosurg 95:190-198, 2001

13. Li YM, Suki D, Hess K, Sawaya R: The influence of maximum safe resection of glioblastoma on survival in 1229 patients: Can we do better than gross-total resection? J Neurosurg 124:977-988, 2016

14. McGirt MJ, Mukherjee D, Chaichana KL, Than KD, Weingart JD, Quinones-Hinojosa A: Association of surgically acquired motor and language deficits on overall survival after resection of glioblastoma multiforme. Neurosurgery 65:463-470, 2009

15. Missios S, Bekelis K, Barnett GH: Renaissance of laser interstitial thermal ablation. Neurosurg Focus 38(3):E13, 2015

16. Mohammadi AM, Hawasli AH, Rodriguez A, Schroeder JL, Laxton AW, Elson P, et al: The role of laser interstitial thermal therapy in enhancing progression-free survival of difficult-to-access high-grade gliomas: a multicenter study. Cancer Med 3:971-979, 2014

17. Montemurro N, Perrini P, Blanco MO, Vannozzi R: Second surgery for recurrent glioblastoma: A concise overview of the current literature. Clin Neurol Neurosurg 142:60-64, 2016

18. Nakagawa M, Matsumoto K, Higashi H, Furuta T, Ohmoto T: Acute effects of interstitial hyperthermia on normal monkey brain-magnetic resonance imaging appearance and effects on blood-brain barrier. Neurol Med Chir (Tokyo) 34:668675,1994

19. Oppenlander ME, Wolf AB, Snyder LA, Bina R, Wilson JR, Coons SW, et al: An extent of resection threshold for recurrent glioblastoma and its risk for neurological morbidity. J Neurosurg 120:846-853, 2014

20. Park CK, Kim JH, Nam DH, Kim CY, Chung SB, Kim YH, et al: A practical scoring system to determine whether to proceed with surgical resection in recurrent glioblastoma. Neuro Oncol 15:1096-1101, 2013

21. Park JK, Hodges T, Arko L, Shen M, Dello Iacono D, McNabb A, et al: Scale to predict survival after surgery for recurrent glioblastoma multiforme. J Clin Oncol 28:38383843,2010

22. Sakai T, Fujishima I, Sugiyama K, Ryu H, Uemura K: Interstitial laserthermia in neurosurgery. J Clin Laser Med Surg 10:37-40, 1992

23. Sanai N, Polley MY, McDermott MW, Parsa AT, Berger MS: An extent of resection threshold for newly diagnosed glioblastomas. J Neurosurg 115:3-8, 2011

24. Schwabe B, Kahn T, Harth T, Ulrich F, Schwarzmaier HJ: Laser-induced thermal lesions in the human brain: short- and long-term appearance on MRI. J Comput Assist Tomogr 21:818-825, 1997

25. Simpson JR, Horton J, Scott C, Curran WJ, Rubin P, Fischbach J, et al: Influence of location and extent of surgical re- 
section on survival of patients with glioblastoma multiforme: results of three consecutive Radiation Therapy Oncology Group (RTOG) clinical trials. Int J Radiat Oncol Biol Phys 26:239-244, 1993

26. Stupp R, Mason WP, van den Bent MJ, Weller M, Fisher $\mathrm{B}$, Taphoorn MJ, et al: Radiotherapy plus concomitant and adjuvant temozolomide for glioblastoma. $\mathbf{N}$ Engl J Med 352:987-996, 2005

27. Vuorinen V, Hinkka S, Färkkilä M, Jääskeläinen J: Debulking or biopsy of malignant glioma in elderly people-a randomised study. Acta Neurochir (Wien) 145:5-10, 2003

28. Walbert T, Mikkelsen T: Recurrent high-grade glioma: a diagnostic and therapeutic challenge. Expert Rev Neurother 11:509-518, 2011

\section{Disclosures}

The authors report no conflict of interest concerning the materi- als or methods used in this study or the findings specified in this paper.

\section{Author Contributions}

Conception and design: all authors. Acquisition of data: all authors. Analysis and interpretation of data: Thomas, Rao, Prabhu. Drafting the article: Thomas, Rao, Prabhu. Critically revising the article: Thomas, Rao, Prabhu. Reviewed submitted version of manuscript: all authors. Approved the final version of the manuscript on behalf of all authors: Thomas. Statistical analysis: Thomas. Administrative/technical/material support: Rao, Prabhu. Study supervision: Rao, Prabhu.

\section{Correspondence}

Jonathan Thomas, Department of Neurosurgery, MD Anderson Cancer Center, 1400 Holcombe Blvd., Unit 442, Houston, TX 77030.email: thomjona@gmail.com. 